\section{Towards risk-stratified induction regimens}

Head and neck squamous cell carcinoma (HNSCC) mortality is $\sim 40 \%$, despite the fact that only $\sim 5 \%$ of patients have metastatic disease at presentation. Hence, improved treatments are needed, particularly for those with locally advanced disease (LAHNSCC), with new data indicating the promise of risk-stratified induction therapy.

In a phase II trial, patients with LA-HNSCC of the oropharynx, oral cavity, nasopharynx, hypopharynx or larynx (T0-4N2b-2c/3M0) received either paclitaxel, carboplatin and the anti-EGFR antibody cetuximab (PCC) or cetuximab, docetaxel, cisplatin and 5-fluorouracil (C-TPF) before local treatment. The 68 patients in each group were stratified into low-risk or higher-risk subgroups according to human papillomavirus (HPV) status and T stage.

Overall, 2-year progression-free survival (PFS) was similar between the treatment arms and, with C-TPF, between the risk groups (88-89\% in each comparison). By contrast, 2-year PFS with PCC was greater in patients with low-risk disease than in those with higher-risk disease (96\% versus 67\%). Thus, PCC and C-TPF might be the preferable induction therapies for low-risk $\left(\mathrm{HPV}^{+} \mathrm{T} 0-3\right.$ or $\left.\mathrm{HPV}^{-} \mathrm{T} 0-2\right)$ and higherrisk $\left(\mathrm{HPV}^{+} \mathrm{T} 4\right.$ or $\left.\mathrm{HPV}^{-} \mathrm{T} 3-4\right)$ disease, respectively. Notably, in comparison with historical cohorts from the RTOG-0129 trial of concurrent chemoradiotherapy without induction therapy, 2-year PFS was improved by $>20 \%$ with PCC in the low-risk group and with C-TPF in the higher-risk group.

In general, PCC and C-TPF had similar safety profiles; although, statistically significant differences in the rates of grade 3-4 skin rash (35\% versus $3 \%$ ), nausea ( $9 \%$ versus $25 \%$ ), hypomagnesaemia ( $1.5 \%$ versus $7.4 \%$ ) and neutropenia (22\% versus $30 \%$ ) were observed.

Further research is needed to establish the optimal approach to risk-stratified induction therapy. More importantly, the true value of induction therapy for LA-HNSCC remains controversial and needs to be clarified.

David Killock

ORIGINAL ARTICLE Haddad, R. I. et al. Weekly paclitaxel, carboplatin, cetuximab, and cetuximab, docetaxel, cisplatin, and fluorouracil, followed by local therapy in previously untreated, locally advanced head and neck squamous cell carcinoma. Ann. Oncol. https://doi.org/10.1093/annonc/ mdy549 (2018)

\title{
SARCOMA
}

\section{Desmoid tumours stalled by sorafenib}

Systemic treatments for desmoid tumours (also known as aggressive fibromatosis) include hormonal blockade, cytotoxic chemotherapy and tyrosine-kinase inhibitors, although response rates to these treatments are varied and no standard-of-care therapy has been defined. A prospective study now shows that sorafenib can slow the progression of this disease.

In a double-blind phase III trial, 87 patients with progressive, symptomatic or recurrent desmoid tumours were randomly assigned to treatment with sorafenib $(n=50)$ or matched placebo $(n=37)$. The primary end point was investigator-assessed progression-free survival (PFS) according to the Response Evaluation Criteria in Solid Tumors (RECIST).

At a median follow-up duration of 27.1 months, the median PFS was not reached in the sorafenib group versus 11.3 months in the placebo group; the estimated 2-year PFS was $81 \%$ versus $36 \%$ (HR 0.13; 95\% Cl 0.05-0.31; $P<0.001$ ). These data indicate an $87 \%$ reduction in the risk of progression or death with sorafenib.

Notably, the benefit of sorafenib did not seem to be dependent on achieving an objective response (objective response rate of $33 \%$ compared with $20 \%$ in the placebo group). Interestingly, $167 \mathrm{MRI}$ scans from 11 patients were analysed to compare changes in tumour dimensions (according to RECIST) with changes in total tumour volume and in MRI T2-weighted signal intensity (which indicates a change from a cellular tumour to a collagenous scar). The results indicate that the latter measures might be more efficient than RECIST in assessing treatment efficacy in patients with desmoid tumours.

Grade 3-4 adverse events occurred in $47 \%$ of patients in the sorafenib group and in $25 \%$ of patients in the placebo group. With sorafenib, the most frequent adverse events were grade $1-2$ rash (73\%), fatigue (67\%), hypertension (55\%) and diarrhoea (51\%). In summary, sorafenib seems to effectively slow the progression of desmoid tumours.

\section{Katharine H. Wrighton}

ORIGINAL ARTICLE Gounder, M. M. et al. Sorafenib for advanced and refractory desmoid tumors. N. Engl.J. Med. 379, 2417-2428 (2018)

\section{Rectal cancer - not a waiting game?}

Rectal cancer surgery, even by gold standard total mesorectal resection (TME), is invasive and carries a risk of complications and morbidities. With the adoption of neoadjuvant chemoradiotherapy, a watchful waiting (WW) approach has been proposed for patients with a clinical complete response (cCR) in order to delay or entirely avoid surgery. The initial results with this strategy have been promising, but new data from a retrospective study from the Memorial Sloan Kettering Cancer Center (New York, USA) raise concerns.

This case-series involved 113 patients with a cCR to neoadjuvant therapy who were subsequently managed through WW. Twenty-two of these patients had local regrowths, all of which were detected through routine surveillance $(72 \%$ within 1 year of cCR), with 20 patients ( $91 \%$ ) having pelvic disease control after salvage surgery. Notably, however, patients with local relapse had a markedly higher risk of distant metastasis (36\% vs $1 \%$; $P<0.001$ ). Moreover, no pelvic recurrences occurred in a control group comprising 136 patients who underwent TME and had a confirmed pathological complete response to neoadjuvant therapy, and only 5 (4\%) of these patients developed metastases.

These differences in oncological control were reflected in the survival data. At 5 years, disease-free survival was $75 \%$ in the WW group versus $92 \%$ in the control group, disease-specific survival was $90 \%$ versus $98 \%$ and overall survival was $73 \%$ versus $94 \%$.

One must be cognizant, however, of the selection and recall biases that are inherent in retrospective studies. Of note, patients in the WW group were older (median age of 67.2 years versus 57.3 years; $P<0.001$ ) and had cancers located closer to the anal verge (median $5.5 \mathrm{~cm}$ versus $7.0 \mathrm{~cm}$ ), which might reflect differences in tumour biology.

Perhaps improved approaches to patient selection for WW are required that ensure a very low risk of recurrence. Importantly, the risks of WW should be weighed against the benefits of organ preservation and reduced morbidity. Notwithstanding, randomized clinical trials are needed to clarify the role of WW in patients with rectal cancer.

David Killock

ORIGINAL ARTICLE Smith, J. J. et al. Assessment of a watch-and-wait strategy for rectal cancer in patients with a complete response after neoadjuvant therapy. JAMA Oncol. https://doi.org/10.1001/jamaoncol.2018.5896 (2019) 